J. Austral. Math. Soc. 19 (Series A) (1975), 225-237.

\title{
GENERALIZED BOOLEAN LATTICES
}

Dedicated to the memory of Hanna Neumann

RICHARD D. BYRD, ROBERTO A. MENA, and LINDA A. TROY

(Received 11 June 1973)

Communicated by G. B. Preston

\section{Introduction}

Hashimoto (1952; Theorems 8.3 and 8.5 ) proved the following theorems:

THEOREM A. If $L$ is a distributive lattice, then there exists a generalized Boolean algebra $L_{r}$ and an isomorphism from the lattice of all congruence relations of $L$ onto the lattice of all congruence relations of $L_{r}$.

THEOREM B. Any distributive lattice $L$ is isomorphic with a sublattice of a relatively comolemented distributive lattice $L_{r}^{*}$ such that (1) the lattice of congruence relations on $L_{r}^{*}$ is isomorphic with that on $L$ and (2) the length of the closed interval $[a, b]$ in $L$ is equal to that of $[a, b]$ regarded as an interval in $L_{r}^{*}$.

It has been noted that Hashimoto's proofs are somewhat difficult to follow and are proved with the apparatus of topology; hence these purely lattice theoretic theorems are not placed in their most natural setting. In 1958 Grätzer and Schmidt (1958; Theorem 1) asserted the following generalization of the Hashimoto theorems:

To any distributive lattice $L$ there exists a generalized Boolean algebra $B$ having the properties:

(1) $L$ is a sublattice of $B$;

(2) the lattice of all congruence relations of $L$ is isomorphic to the lattice of all congruence relations of $B$;

(3) if the interval $[a, b]$ of $L$ is of finite length, then $[a, b]$ has the same length as an interval of $B$.

In this note we give a counterexample to (2). In Section 2 we construct an ideal $E$ of $B$ and prove that the lattice of all congruence relations of $L$ is isomorphic to the lattice of all congruence relations of $E$ (Corollary 2.11). We prove that $E=B$ if and only if $0 \in L$ (Theorem 2.4); otherwise, $E$ is a maximal ideal of $B$ (Corollary 2.5). Our example shows that in general $L$ cannot be embedded into $E$. The construction of $E$ is algebraic and, moreover, we prove that $E$ is unique up to isomorphism (Corollary 2.12). Thus we strengthen Theorem A. 
Our example also shows that Grätzer (1971; Lemma 5, page 104)) is incorrect (Lemma 5 is in essence (2) above). We prove in Section 3 (also, see Corollary 4.3) that a necessary and sufficient condition that this lemma be true is that the lattice $L$ has a smallest element.

In Section 4 we investigate distributive lattices $K$ and $L$, where $L$ is a sublattice of $K$ and each congruence of $L$ has a unique extension to $K$. In this case we prove that there is a generalized Boolean lattice $B$ that is $R$-generated by $L$ and contains $K$ as a sublattice (Theorem 4.5). From this result we obtain several interesting corollaries; one of which asserts that the lattice $L_{r}^{*}$ of Theorem B is unique up to isomorphism.

In Section 5 we give the example.

Throughout this note $L$ will denote a distributive lattice, $\mathscr{C}(L)$ will denote the lattice of all congruence relations on $L, \mathscr{T}(L)$ will denote the lattice of all ideals of $L, \mathbb{N}$ will denote the set of natural numbers, $\mathbb{Z}$ will denote the set integers, $\square$ will denote the empty set, and $X \backslash Y$ will denote the set of elements that belong to the set $X$ but not to the set $Y$. Unless otherwise stated, isomorphism will mean a homomorphism that is one-to-one (not necessarily onto). For the standard results and definitions concerning lattices, the reader is referred to Grätzer (1971) in particular, to Sections 9 and 10 of Chapter 2.

\section{Evenly generated ideals}

Throughout this section let $B$ be a generalized Boolean lattice and let $L$ be a sublattice of $B$ that generates $B$, that is, the smallest subring of $B$ that contains $L$ is $B$.

Lemma 2.1 (i) $B=\left\{a_{1}+\cdots+a_{n} \mid n \in \mathbb{N}\right.$ and $\left.a_{1}, \cdots, a_{n} \in L\right\}$.

(ii) If $T$ is a sublattice of $L$ and $x \in B$ such that $x=a_{1}+\cdots+a_{n}(n \in \mathbb{N})$, where $a_{1}, \cdots, a_{n} \in T$, then $x=b_{1}+\cdots+b_{n}$, where $b_{1}, \cdots, b_{n} \in T$ and $b_{1} \leqq \cdots \leqq b_{n}$.

The proof of this lemma is similar to the proof of Grätzer (1971; Lemma 3, page 102) and will be omitted.

COROLlARY 2.2. If T is a sublattice of $L$ and $x \in B$ such that $x=a_{1}+\cdots+a_{2 n-1}$ $(n \in \mathbb{N})$, where $a_{1}, \cdots, a_{2 n-1} \in T$, then $x \geqq a$ for some $a$ in $T$.

Proof. By the lemma, $x=b_{1}+\cdots+b_{2 n-1}$, where $b_{1}, \cdots, b_{2 n-1} \in T$ and $b_{1} \leqq \cdots \leqq b_{2 n-1}$. Thus $x \geqq x \wedge b_{1}=x b_{1}=b_{1}$, since $2 n-1$ is odd and $b_{1} b_{j}=b_{1}$ for each $j$.

Lemma 2.3. Let $T$ be an ideal of $L$ and $E_{T}=\{x \mid x \in B$ and there exists $a_{1}, \cdots, a_{2 n} \in T(n \in \mathbb{N})$ such that $\left.x=a_{1}+\cdots+a_{2 n}\right\}$. Then

(i) $E_{T}$ is an ideal of $B$;

(ii) $E_{T}=E_{L} \cap(T]_{B}$, where $(T]_{B}$ denotes the ideal of $B$ generated by $T$; 
(iii) $E_{r}=\left\{x \mid x \in B\right.$ and there exists $a_{1}, \cdots, a_{2 n} \in T$ such that $a_{1} \leqq \cdots \leqq a_{2 n}$ and $\left.x=a_{1}+\cdots+a_{2 n}\right\}$.

The proof of this lemma is straightforward and will be omitted. We shall call the ideal $E_{T}$ of this lemma the ideal of $B$ evenly generated by $T$. Also for the remainder of this note we shall denote $E_{L}$ simply by $E$.

In Corollary 2.2, if $T=L$ and $x$ in $B$ has a representation as a sum of an odd number of elements from $L$, then $x$ exceeds an element of $L$. The next theorem shows that if $L$ does not contain the zero of $B$ and $x$ in $B$ has a representation as a sum of an even number of elements of $L$, then $x$ does not exceed an element of $L$. Also, if $x=a_{1}+\cdots+a_{n}$, where $a_{1}, \cdots, a_{n} \in L$, then $x \in\left(a_{1} \vee \cdots \vee a_{n}\right]_{B}$ and so each element of $B$ is exceeded by an element of $L$. We say that $L R$-generates $B$ if $L$ generates $B$ and if $L$ has a least element, then it is the zero of $B$. (The definition of $R$-generates given in Grätzer(1971; page 102) also required that if $L$ has a largest element, then it must be the largest element of $B$.) If $L$ does not have a smallest element, then the definitions of $R$-generates and generates coincide.

THEOREM 2.4. If $L$ generates $B$, then the following assertions are equivalent:

(i) $0 \in L$.

(ii) $L \cap E \neq \square$.

(iii) $L \subseteq E$.

(iv) $E=B$.

Proof. (i) implies (ii) is immediate as $0 \in L \cap E$.

(ii) implies (iii). Let $a \in L$ and $b \in L \cap E$. Then $a+b \in E$ and hence $a=a+b+b \in E$.

(iii) implies (iv) is obvious as $E$ is an ideal of $B$.

(iv) implies (i). If $b \in L$, then $b=a_{1}+\cdots+a_{2 n}$, where $a_{1}, \cdots, a_{2 n} \in L$ and $a_{1} \leqq \cdots \leqq a_{2 n}$. Then $b \wedge a_{1} \in L$ and $b \wedge a_{1}=b a_{1}=a_{1}+\cdots+a_{1}$, where there are $2 n$ summands. Therefore $b a_{1}=0$ and so $0 \in L$.

Corollary 2.5. If $0 \notin L$, then the index of $E$ in $B$ is two and hence $E$ is a maximal ideal of $B$. Moreover, $L$ is a sublattice of the relatively complemented lattice $B \backslash E$ and if $M$ is an ideal of $B$ such that $M \cap L=\square$, then $M \subseteq E$.

Proof. By the theorem, $E \neq B$ and $L \subseteq B \backslash E$. If $x \in B \backslash E$, then $x=a_{1}+\cdots+$ $a_{2 n-1}$, where $a_{1}, \cdots, a_{2 n-1} \in L$. If $n=1, x=a_{1}$ and if $n>1, a_{1}+\cdots+a_{2 n-2} \in E$. Thus $E+x=E+a$ for some $a \in L$. If $a, b \in L$, then $a+b \in E$ and so $E+a$ $=E+b$. Therefore the index of $E$ in $B$ is two.

Since $E$ is an ideal of $B$ and $B$ is a generalized Boolean lattice, $B \backslash E$ is relatively complemented. If $M \in \mathscr{I}(B) \mid \mathscr{I}(E)$, then $M \cap L \neq \square$ by Corollary 2.2. 
LEMMA 2.6. Let $\theta \in \mathscr{C}(L)$ and $I_{\theta}$ be the ideal of $B$ generated by $\{a+b \mid a, b \in L$ and $a \theta b\}$. Then $I_{\theta}=\left\{\sum_{i=1}^{n} a_{i}+b_{i} \mid n \in \mathbb{N}, a_{i}, b_{i} \in L, a_{i} \leqq b_{i}\right.$, and $\left.a_{i} \theta b_{i}\right\}$ and so $I_{\theta} \in \mathscr{I}(E)$.

Proof. For $a, b \in L, a \theta b$ if and only if $a \wedge b \theta a \vee b$. Also, $a+b=$ $a b+a+b+a b=a \wedge b+a \vee b$. Thus, $I_{\theta}$ is the ideal of $B$ generated by $\{a+b \mid a, b \in L, a \leqq b$, and $a \theta b\}$. Let $I=\left\{\sum_{i=1}^{n} a_{i}+b_{i} \mid n \in \mathbb{N}, a_{i}, b_{i} \in L\right.$, $a_{i} \leqq b_{i}$, and $\left.a_{i} \theta b_{i}\right\}$. Then clearly $\{a+b \mid a, b \in L, a \leqq b$, and $a \theta b\} \subseteq I \subseteq I_{\theta}$ and $I$ is closed with respect to addition. To prove that $I$ is an ideal of $B$, it suffices to show that for $x \in I$ and $a \in L, a x \in I$. Let $x=\sum_{i=1}^{n} a_{i}+b_{i}$, where $a_{i}, b_{i} \in L$, $a_{i} \leqq b_{i}$, and $a_{i} \theta b_{i}$. For each $i, a a_{i}=a \wedge a_{i} \leqq a \wedge b_{i}=a b_{i}$ and $a_{i} \theta b_{i}$ implies $a \wedge a_{i} \theta a \wedge b_{i}$. Thus $a x \in I$ and so $I=I_{\theta}$. Clearly $I_{\theta} \in \mathscr{I}(E)$.

Lemma 2.7. Let $I \in \mathscr{I}(E)$ and $\theta_{I}=\{(a, b) \mid a, b \in L$ and $a+b \in I\}$. Then $\theta_{I}$ is a congruence relation of $L$.

Proof. It is easily verified that $\theta_{I}$ is an equivalence relation of $L$ and if $a \theta b$ and $t \in L$, then $a \wedge t \theta b \wedge t$. Now $a \vee t+b \vee t=a+t+a t+b+t+b t=$ $a+b+(a+b) t \in I$. Therefore $a \vee t \theta b \vee t$ and so $\theta_{I} \in \mathscr{C}(L)$.

TheOREM 2.8. The mapping $g$ of $\mathscr{I}(E)$ into $\mathscr{C}(L)$ given by $(I) g=\theta_{I}$ is an isomorphism of $\mathscr{I}(E)$ onto $\mathscr{C}(L)$. The inverse of $g$ is given by $(\theta) g^{-1}=I_{\theta}$.

Proof. By Lemma 2.7, $g$ is a mapping of $\mathscr{I}(E)$ into $\mathscr{C}(L)$. If $\theta \in \mathscr{C}(L)$, then by Lemma 2.6, $I_{\theta} \in \mathscr{I}(E)$. We will show that $\left(I_{\theta}\right) g=\theta_{I_{\theta}}=\theta$. If $a \theta b$, then $a+b \in I_{\theta}$ and so $a \theta_{I_{\theta}} b$. Conversely if $a \theta_{I_{\theta}} b$, then $a+b \in I_{\theta}$ and, without loss of generality, we may assume that $a \leqq b$. Now $a+b \in I_{\theta}$ implies that $a+b=$ $\sum_{i=1}^{n} a_{i}+b_{i}$, where $a_{i}, b_{i} \in L, a_{i} \leqq b_{i}$, and $a_{i} \theta b_{i}$. We induct on $n$. If $n=1$, then $a+b=(a+b) b=a_{1} b+b_{1} b, a_{1} b \leqq b_{1} b$, and $a_{1} b \theta b_{1} b$. Since $b_{1} b \leqq b$, we may assume that $a+b=a_{1}+b_{1}$, where $a_{1} \theta b_{1}$ and $a_{1} \leqq b_{1} \leqq b$. Therefore $0=a_{1}+a_{1}=\left(a_{1}+b_{1}\right) a_{1}=a a_{1}+b a_{1}=a a_{1}+a_{1}$. Hence $a_{1} \leqq a$. Now $a+b=a_{1}+b_{1} \leqq b_{1}$, as $a_{1} \leqq b_{1}$, and so $n=a \vee(a+b) \leqq a \vee b_{1} \leqq a \vee b=b$. Whence $a \vee b_{1}=b$ and $a \vee a_{1}=a$. Thus $a_{1} \theta b_{1}$ implies $a \vee a_{1} \theta a \vee b_{1}$ or $a \theta b$. Next assume that $n>1$. Again $a+b=\sum_{i=1}^{n} a_{i} b+b_{i} b$ and so we may assume that $a_{i} \leqq b_{i} \leqq b$ for each $i$. Let $d=V^{n}{ }_{i=1} b_{i}$. For $1 \leqq k \leqq n, a a_{k}+a_{k}$ $=(a+b) a_{k}=\sum_{i=1, i * k}^{n} a_{i} a_{k}+b_{i} a_{k}$. Now a $\theta_{I_{\theta}} b$ implies that $a a_{k} \theta_{I_{\theta}} a_{k}$ and hence by the inductive hypothesis $a a_{k} \theta a_{k}$. Since $a_{k} \theta b_{k}$, we have $a a_{k} \theta b_{k}$ and so $a \theta a \vee b_{k}$. Also $a d+d=(a+b) d=\sum_{i=1}^{n} a_{i} d+b_{i} d=\sum_{i=1}^{n} a_{i}+b_{i}=a+b$. Hence $a \vee d=a+d+a d=b$. Thus we have $a \theta \vee{ }_{i=1}^{n}\left(a \vee b_{i}\right)$, which is equivalent to $a \theta b$. Therefore $g$ maps $\mathscr{I}(E)$ onto $\mathscr{C}(L)$.

Let $I, J \in \mathscr{I}(E)$ with $I \neq J$ and let $x \in J \backslash I$. Then there exists $a_{1}, \cdots, a_{2 n} \in L$ with $a_{1} \leqq \cdots \leqq a_{2 n}$ and $x=a_{1}+\cdots+a_{2 n}$. Multiplying $x$ respectively by $a_{2}, \cdots, a_{2 n-2}$, we obtain $a_{1}+a_{2}, \cdots, a_{2 n-1}+a_{2 n} \in J$. Hence for some $k,(1 \leqq k \leqq n)$ $a_{2 k-1}+a_{2 k} \in J \backslash I$. Therefore $\left(a_{2 k-1}, a_{2 k}\right) \in \theta_{J} \mid \theta_{I}$ and so $g$ is one-to-one. 
If $I, J \in \mathscr{I}(E)$, then clearly $I \subseteq J$ if and only if $\theta_{I} \subseteq \theta_{J}$. Therefore $g$ is an isomorphism of $\mathscr{I}(E)$ onto $\mathscr{C}(L)$. It is now clear that $(\theta) g^{-1}=I_{\theta}$.

An immediate consequence of this theorem is

CoRollary 2.9. (i) If $\theta \in \mathscr{C}(L)$, then $\theta=\theta_{I_{\theta}}$.

(ii) If $I \in \mathscr{I}(E)$, then $I=I_{\theta_{I}}$.

Corollary 2.10. (Hashimoto, (1972; Theorem 7.2) $\mathscr{I}(E)$ lis isomorphic to $\mathscr{C}(E)$.

Proof. Since $E$ is a generalized Boolean lattice and $E$ generates itself, we have by the theorem that $\mathscr{I}(E)$ is isomorphic to $\mathscr{C}(E)$.

Corollary 2.11. $\mathscr{C}(L)$ is isomorphic to $\mathscr{C}(E)$.

COROLlaRY 2.12. If $D$ is a generalized Boolean lattice such that $\mathscr{C}(L)$ is isomorphic to $\mathscr{C}(D)$, then $D$ is isomorphic to $E$.

Proof. As noted above, $\mathscr{C}(D)$ is isomorphic to $\mathscr{F}(D)$. Hence $\mathscr{F}(D)$ and $\mathscr{I}(E)$ are isomorphic. The compact elements of $\mathscr{I}(E)$ are the principal ideals of $E$, which are isomorphic to $E$. Since compact elements are preserved under isomorphism, it follows that $E$ is isomorphic to $D$.

If $T \in \mathscr{I}(L)$, let $\theta_{T}=\{(a, b) \mid a, b \in L$ and $a \vee t=b \vee t$ for some $t \in T\}$. It is easily verified that $\theta_{T}=\{(a, b) \mid a, b \in L$ and $a \vee b=(a \wedge b) \vee t$ for some $t \in T\}$ and that the mapping that sends $T$ into $\theta_{T}$ is an isomorphism of $\mathscr{I}(L)$ onto a sublattice of $\mathscr{C}(L)$ (Hashimoto (1973; Theorem 5.1)). Moreover, for each $t \in T,[t] \theta_{T}=T$, where $[t] \theta_{T}$ denotes the congruence class of $\theta_{T}$ containing $t$.

CoROLlaRY 2.13. Let $h$ be the mapping of $\mathscr{I}(L)$ into $\mathscr{F}(E)$ given by $(T) h=E_{T}$. Then $h$ is an isomorphism of $\mathscr{I}(L)$ onto a sublattice of $\mathscr{I}(E)$.

Proof. It suffices to show that $E_{T}=I_{\theta_{T}}$. If $s, t \in T$, then $s \theta_{T} t$ and so $s+t \in I_{\theta_{T}}$. It follows that $E_{T}=I_{\theta_{T}}$. Conversely, let $a, b \in L$ with $a \theta_{T} b$ and $a+b$ $\in I_{\theta_{\mathbf{T}}}$. Then $a \vee b=(a \wedge b) \vee t$ for some $t$ in $T$ and so $a+b+a b=a b+t+a b t$. Therefore $a+b=t+a b t$ and since $t, a b t \in T$, we have $a+b \in E_{T}$. Again it follows that $I_{\theta} \subseteq E_{T}$.

We have now obtained the Hashimoto theorems mentioned in the introduction. For let $L$ be a distributive lattice and $B$ be a generalized Boolean lattice $R$-generated by $L$. Then $E=E_{L}$ is a generalized Boolean lattice and $\mathscr{C}(E)$ is isomorphic to $\mathscr{C}(L)$ (Corollary 2.11). Moreover, we have proven that, up to isomorphism, $E$ is unique (Corollary 2.12). If $L$ has a smallest element, then $E=B$ (Theorem 2.4). If $L$ does not have a smallest element, then $L \subseteq B \backslash E$ (Theorem 2.4). Let $K=B \backslash L$. Then $K$ is a prime dual ideal of $B$ since $E$ is a prime ideal of $B$ (Corollary 2.5). Thus $K$ is a relatively complemented distributive lattice, $L$ is a sublattice of $K$, and $K$ does not have a smallest element (Corollary 2.2). 
If we let $E_{K}=\left\{c_{1}+\cdots+c_{2 n} \mid c_{1}, \cdots, c_{2 n} \in K\right\}$, then it is easily verified that $E_{K}=E$. Since $K$ generates $B, \mathscr{C}(K)$ is isomorphic to $\mathscr{C}\left(E_{K}\right)$ (Corollary 2.11) and so $\mathscr{C}(K)$ is isomorphic to $\mathscr{C}(L)$. We will prove in Section 4 that, up to isomorphism, $K$ is unique (see Corollary 4.9 ).

Corollary 2.14. Let $F$ be a maximal ideal of $B$ and $K=B \backslash F$. Then $F=\left\{x \mid x \in B\right.$ and there exists $c_{1}, \cdots, c_{2 n} \in K(n \in \mathbb{N})$ such that $\left.x=c_{1}+\cdots+c_{2 n}\right\}$. Hence $K$ generates $B$ and $\mathscr{C}(K)$ is isomorphic to $\mathscr{C}(F)$.

Proof. Let $E_{K}=\left\{c_{1}+\cdots+c_{2 n} \mid c_{1}, \cdots, c_{2 n} \in K\right\}$. Since $F$ is maximal, $K$ is a dual ideal of $B$ and hence a sublattice of $B$. Let $c, d \in K$. Since $c+d=c \wedge d+c \vee d$, we may assume that $c \leqq d$. Thus $c \wedge(c+d)=0$. Thus $c+d \in F$, as $F$ is prime, and it follows that $E_{K} \subseteq F$. If $x \in F$ and $c \in K$, $x+c \in F+c \subseteq K$ and so $x \in K+c \subseteq E_{K}$. Therefore $F=E_{K}$ and $K$ generates $B$. Since $E_{K}$ is the ideal of $B$ evenly generated by $K$ we have by Corollary 2.11 that $\mathscr{C}(K)$ is isomorphic to $\mathscr{C}(F)$.

Observe that $K$ is a relatively complemented lattice, but in general does not have a smallest element. Hence in general $\mathscr{I}(K)$ and $\mathscr{C}(K)$ are not isomorphic. To this end we prove

THEOREM 2.15. Let $F$ be a maximal ideal of $B$ and $K=B \backslash F$. Then the following are equivalent:

(i) $K$ is a generalized Boolean lattice.

(ii) $K$ has a smallest element.

(iii) $K$ is isomorphic to $F$.

(iv) There exists $c \in K$ such that $c F=\{0\}$.

(v) There exists an atom of $B$ in $K$.

(vi) $F$ is a direct summand of $B$.

Proof. (i) implies (ii) is trivial.

(ii) implies (iii). As noted above, $K$ is always a relatively complemented lattice and so by (ii), $K$ is a generalized Boolean lattice. Thus by Corollary 2.12, $K$ is isomorphic to $F$.

(iii) implies (iv). If $K$ is ismorphic to $F, K$ has a least element $c_{0}$. Now $F$ is generated by $\{c+d \mid c, d \in K$ and $c \leqq d\}$. For $c, d \in K$ with $d \geqq c, c \geqq c_{0}$ and so $0 \leqq c_{0} \wedge(c+d) \leqq c \wedge(c+d)=0$. It follows that $c_{0} F=\{0\}$.

(iv) implies (v). Let $c \in K$ such that $c F=\{0\}$. Let $x \in B$ with $0 \leqq x \leqq c$. If $x \in F$, then $0=c x=x$. If $x \in K$, then $c+x \in F$ and $0=(c+x) c=c+x$. Therefore $x=c$ and hence $c$ is an atom of $B$.

(v) implies (vi). Let $c$ be an atom of $B$ in $K$. Then $(c]_{B} \cap F=\{0\}$. Since $F$ is a maximal ideal of $B, F+(c]_{B}=B$ and so $F$ is a direct summand of $B$.

(vi) implies (i). Suppose that $B$ is the direct sum of $F$ and $M$, where $M$ is an ideal of $B$. Since the index of $F$ in $B$ is two, it follows that $M=\{0, c\}$ for 
some $c \in K$. If $x \in K$, then $x \wedge c=c$ or $x \wedge c=0$. Since $F$ is prime, $x \wedge c \neq 0$. Therefore $c$ is the smallest element of $K$ and hence $K$ is a generalized Boolean lattice.

In concluding this section, we note that Theorem 2.15 exemplifies a reason for requiring in the definition of $L R$-generates $B$ that if $L$ has a smallest element, it must be the zero of $B$.

\section{Extending congruences}

Throughout this section let $L$ denote a lattice without a smallest element, $L_{0}$ denote the lattice $L$ with a smallest element 0 adjoined, and let $\mathscr{C}_{0}(L)=\{\theta \mid \theta \in \mathscr{C}(L)$ and $L / \theta$ has a least element $\}$. J. Hashimoto (1973; Theorem 5.1) proved that $\mathscr{C}_{0}(L)$ is a dual ideal of $\mathscr{C}(L)$. The hypothesis that $L$ is distributive is not needed for the proofs of Lemma 3.1, Corollary 3.2, and Theorems 3.3 and 3.4 .

LEMMA 3.1. If $\theta$ is a congruence relation of $L$, then $\theta$ can be extended to a congruence relation of $L_{0}$. Moreover,

(i) if $\theta \notin \mathscr{C}_{0}(L)$, then $\theta$ has a unique extension to $L_{0}$;

(ii) if $\theta \in \mathscr{C}_{0}(L)$, then $\theta$ has exactly two extensions to $L_{0}$.

Proof. If $\theta_{0}=\theta \cup\{(0,0)\}$, then $\theta_{0}$ is a congruence relation of $L_{0}$ that extends $\theta$. If $\theta \notin \mathscr{C}_{0}(L)$, then it is easily verified that $\theta_{0}$ is the only extension of $\theta$. If $\theta \in \mathscr{C}_{0}(L)$ and $T$ is the smallest element of $L / \theta$, then let $\theta_{1}=\theta \cup(T \times\{0\})$ $\cup(\{0\} \times T) \cup\{(0,0)\}$. Then $\theta_{1}$ is a congruence relation of $L_{0}$ that extends $\theta$ and $\theta_{1}>\theta_{0}$. Again it is easily verified that these are the only extensions of $\theta$ in $\mathscr{C}\left(L_{0}\right)$.

COROLlary 3.2. A congruence relation $\theta$ of $L$ has a unique extension to a congruence relation of $L_{0}$ if and only if $\theta \notin \mathscr{C}\left(L_{0}\right)$.

If $T$ is an ideal of $L$, we observed in Section 2 that $\theta_{T} \in \mathscr{C}(L)$ and trivially $T$ is the least element of $L / \theta_{T}$. Thus by the theorem, $\theta_{T}$ has two extensions to $L_{0}$. It is evident that if $B$ is a generalized Boolean lattice $R$-generated by $L_{0}$, then $B$ is also $R$-generated by $L$. We note that Lemma 5 (Grätzer (1971; page 104)) is valid if the lattice has a smallest element and hence each congruence of $L_{0}$ has a unique extension to $B$, but Lemma 3.1 shows that each element of $\mathscr{C}_{0}(L)$ has two extensions to $B$. Since $\mathscr{C}(L)$ is isomorphic to $\mathscr{C}(E)$ and $\omega\left(L_{0}\right)$ is isomorphic to $\mathscr{C}(B)$, this raises the following question: which congruences of $E$ have two extensions to $B$ ? (By Grätzer (1971; Theorem 6, page 90) each congruence of $E$ has at least one extension to $B$.) In this section we give an answer to this question.

Let $f_{0}$ and ${ }^{\prime}$, be the mappings of $\mathscr{C}(L)$ into $\mathscr{C}\left(L_{0}\right)$ given by

$$
\theta f_{0}=\theta_{0}
$$


and

$$
\theta f_{1}= \begin{cases}\theta_{1} & \text { if } \theta \in \mathscr{C}_{0}(L) \\ \theta f_{0} & \text { otherwise, }\end{cases}
$$

where $\theta \in \mathscr{C}(L)$ and $\theta_{0}$ and $\theta_{1}$ are given above.

THEOREM 3.3. (i) $f_{0}$ is an isomorphism of $\mathscr{C}(L)$ into $\mathscr{C}\left(L_{0}\right)$.

(ii) $f_{1}$ is a one-to-one inclusion preserving mapping of $\mathscr{C}(L)$ into $\mathscr{C}\left(L_{0}\right)$ and $f_{1} \mid \mathscr{C}_{0}(L)$ is an isomorphism.

PRoof. The verification of (i) and that $f_{1}$ is a one-to-one inclusion preserving mapping is straightforward and will be omitted. Let $\theta, \Psi \in \mathscr{C}_{0}(L)$ and let $S$ and $T$ denote the smallest elements of $\theta$ and $\Psi$ respectively. Then $S \cap T$ is the smallest element of $\theta \wedge \Psi$ (Hashimoto (1952; Lemma 5.1)). Then

$$
\begin{aligned}
\theta_{1} \wedge \Psi_{1} & =(\theta \cup(S \times\{0\}) \cup(\{0\} \times S) \cup\{(0,0)\} \cap(\Psi \cup(T \times\{0\}) \cup(\{0\} \times T) \\
& =(\theta \cap \Psi) \cup((S \cap T) \times\{0\}) \cup(\{0\} \times(S \cap T)) \cup\{(0,0)\} \\
& =(\theta \wedge \Psi)_{1} .
\end{aligned}
$$

Since $f_{1}$ is inclusion preserving $\theta_{1} \vee \Psi_{1} \subseteq(\theta \vee \Psi)_{1}$. Let $(a, b) \in(\theta \vee \Psi)_{1}$ and $U$ be the least element of $L /(\theta \vee \Psi)$. If $(a, b) \in \theta \vee \Psi$, then clearly $(a, b) \in \theta_{1} \vee \Psi_{1}$. If $(a, b) \in(U \times\{0\}) \cup(\{0\} \times U)$, then, since $(\theta \vee \Psi)_{1}$ is symmetric, we assume that $a \in U$ and $b=0$. Now $a \in U$ implies that there exists $b_{0}, \cdots, b_{m} \in L$ such that $a=b_{0}, b_{m} \in T$, and $\left(b_{i}, b_{i+1}\right) \in \theta \cup \Psi$. Hence $\left(a, b_{m}\right) \in \theta_{1} \vee \Psi_{1}$. Since $T \subseteq U$ (Hashimoto (1952; Lemma 5.1)). $\left(b_{m}, 0\right) \in \Psi_{1}$. Therefore $(a, 0) \in \theta_{1} \vee \Psi_{1}$. Trivially, if $a=b=0,(a, b) \in \theta_{1} \vee \Psi_{1}$.

We now describe the lattice $\mathscr{C}\left(L_{0}\right)$ in terms of $\mathscr{C}(L)$.

TheOREM 3.4. $\mathscr{C}\left(L_{0}\right)$ is the disjoint union of $(\mathscr{C}(L)) f_{0}$ and $\left(\mathscr{C}_{0}(L)\right) f_{1}$. Moreover, $\left(\mathscr{C}_{0}(L)\right) f_{1}$ is a prime dual ideal of $\mathscr{C}\left(L_{0}\right)$ and for $\theta \in \mathscr{C}\left({ }_{L}\right)$ and $\Psi \in \mathscr{C}_{0}(L)$,

and

$$
\theta_{0} \wedge \Psi_{1}=(\theta \wedge \Psi)_{0}
$$

$$
\theta_{0} \vee \Psi_{1}=(\theta \vee \Psi)_{1}
$$

Proof. Using Lemma 3.1, Theorem 3.3, and the fact that $L$ is a sublattice of $L_{0}$, it is easily verified that $\mathscr{C}\left(L_{0}\right)$ is the disjoint union of $(\mathscr{C}(L)) f_{0}$ and $\left(\mathscr{C}_{0}(L)\right) f_{1}$ and that $\left(\mathscr{C}_{0}(L)\right) f_{1}$ is a prime dual ideal of $\mathscr{C}\left(L_{0}\right)$.

Let $\theta \in \mathscr{C}(L), \Psi \in \mathscr{C}_{0}(L)$, and $T$ be the least element of $L / \Psi$. Then

$$
\begin{aligned}
\theta_{0} \wedge \Psi_{1} & =(\theta \cup\{(0,0)\}) \cap(\Psi \cup(T \times\{0\}) \cup(\{0\} \times T) \cup\{(0,0)\}) \\
& =(\theta \cap \Psi) \cup\{(0,0)\} \\
& =(\theta \wedge \Psi)_{0} .
\end{aligned}
$$


Since $f_{1}$ is inclusion preserving $\theta_{0} \subseteq(\theta \vee \Psi)_{1}$ and $\Psi_{1} \subseteq(\theta \vee \Psi)_{1}$. Therefore $\theta_{0} \vee \Psi_{1} \subseteq(\theta \vee \Psi)_{1}$. Let $(a, b) \in(\theta \vee \Psi)_{1}$ and let $U$ be the least element of $L /(\theta \vee \Psi)$. If $(a, b) \in \theta \vee \Psi$, then clearly $(a, b) \in \theta_{0} \vee \Psi_{1}$. Suppose that $(a, b) \in U \times\{0\}$. Then there exists $b_{0}, \cdots, b_{m} \in L$ such that $a=b_{0}, b_{m} \in T$, and $\left(b_{i}, b_{i+1}\right) \in \theta \cup \Psi$. As above, $\left(a, b_{m}\right) \in \theta_{0} \vee \Psi_{1}$ and $\left(b_{m}, 0\right) \in \Psi_{1}$. Hence $(a, 0)$ $\in \theta_{0} \vee \Psi_{1}$. It follows that $(\theta \vee \Psi)_{1} \subseteq \theta_{0} \vee \Psi_{1}$.

For the remainder of this section, let $B$ be a generalized Boolean lattice $R$-generated by $L$ and $E$ be the ideal of $B$ evenly generated by $L$. Then by Corollary $2.5, E$ is a maximal ideal of $B$. Obviously $B$ is also $R$-generated by $L_{0}$ and by Theorem 2.4 the ideal of $B$ evenly generated by $L_{0}$ is $B$. Let $p$ be the isomorphism of $\mathscr{C}(L)$ onto $\mathscr{I}(E)$ given by $(\theta) p=I_{\theta}$ ( $p$ is the inverse of the isomorphism $g$ of Theorem 2.8), let $p_{0}$ be the corresponding isomorphism of $\mathscr{C}\left(L_{0}\right)$ onto $\mathscr{I}(B)$, let $\mathbf{t}$ be the inclusion mapping of $\mathscr{I}(E)$ into $\mathscr{I}(B)$, and let $f_{0}$ be as above.

LEMma 3.5. If $\theta \in \mathscr{C}(L)$, then $(\theta) p \imath=(\theta) f_{0} p_{0}$.

The proof of this lemma is routine and hence will be omitted.

Lemma 3.6. Let $F$ be a maximal ideal of $B$ and $I$ be an ideal of $F$. Then there are at most two ideals of $B$ whose intersections with $F$ is $I$.

Proof. Let $M, N \in \mathscr{I}(B) \mid \mathscr{I}(F)$ such that $M \cap F=N \cap F=I$. Since $F$ is maximal, we have $M=M \wedge B=M \wedge(F \vee N)=(M \wedge F) \vee(M \wedge N)=$ $I \vee(M \wedge N) \subseteq N$. Dually $N_{s} \subseteq M$. Trivially $I$ is the only ideal of $F$ whose intersection with $F$ is $I$.

THEOREM 3.7. Let $I$ be an ideal of $E$. Then there exists $M \in \mathscr{I}(B) \backslash \mathscr{I}(E)$ such that $M \cap E=I$ if and only if $(I) p^{-1} \in \mathscr{C}_{0}(L)$.

Proof. Let $M \in \mathscr{I}(B) \backslash \mathscr{I}(E)$ such that $M \cap E=I, T=M \cap L$, and $\theta=I p^{-1}$. Then $T \in \mathscr{I}(L)$ and to prove that $\theta \in \mathscr{C}_{0}(L)$, it suffices to show that $T \in L / \theta$. If $a, b \in T$, then $a+b \in M \cap E$ and hence by Lemma $2.7, a \theta b$. Conversely let $a \in T$ and $b \in[a] \theta$. Then $a+b \in I \subseteq M$. Thus we have $b=a+a+b \in M$. Therefore $b \in M \cap L=T$ and so $T \in L / \theta$. Since $T \in \mathscr{I}(L)$, it is the smallest element of $L / \theta$. Hence $\theta \in \mathscr{C}_{0}(L)$.

Next suppose that $\theta=I p^{-1} \in \mathscr{C}_{0}(L)$. Then $\theta_{0}=\theta f_{1} \cap((L \times L) \cup\{(0,0)\})$ $=\theta f_{1} \cap(L \times L) f_{0}$. By Theorem 3.4, $\theta f_{1} \cap(L \times L) f_{0}=\theta f_{0}$ and by Lemma 3.5, $I=\theta p l=\theta f_{0} g_{0}=\theta f_{1} g_{0} \cap(L \times L) f_{0} g_{0}=\theta f_{1} g_{0} \cap E$. Now $\theta f_{1} g_{0} \in \mathscr{I}(B)$ and if $T$ is the smallest element of $L / \theta$, then $T \subseteq \theta f_{1} g_{0}$. Therefore $\theta f_{1} g_{0} \notin \mathscr{I}(E)$.

It follows from this theorem that a congruence of $E$ which has exactly two extensions to $B$ is induced (see the discussion after Corollary 2.12) by an element from $\left(\mathscr{C}_{0}(L)\right) p$.

\section{Extension Property}

Let $K$ be a distributive lattice. We say that $K$ has the extension property (EP) over $L$ if $L$ is a sublattice of $K$ and each congruence of $L$ can be uniquely 
extended to a congruence of $K$. If $L$ is a sublattice of $K$, then $K$ has (EP) over $L$ if and only if the mapping of $\mathscr{C}(K)$ into $\mathscr{C}(L)$, which sends $\chi$ onto $\chi \cap(L \times L)$, is one-to-one. (It is well known that this mapping is onto (Grätzer (1971; Theorem 6 , page 90)). The next lemma is immediate from the definition.

LeMMA 4.1. Let $K$ be a distributive lattice and $M$ and $L$ be sublattices of $K$ such that $L \subseteq M$. Then $K$ has $(E P)$ over $L$ if and only if $K$ has $(E P)$ over $M$ and $M$ has $(E P)$ over $L$.

THEOREM 4.2. Let $B$ be a generalized Boolean lattice R-generated by $L$.

(i) If $0 \in L$ and $K$ is a sublattice of $B$ that contains $L$, then $K$ has (EP) over $L$.

(ii) If $0 \notin L$ and $K$ is a sublattice of $B \backslash E$ that contains $L$, then $K$ has (EP) over $L$.

Proof. Let $K$ be as either in (i) or (ii), $\theta \in \mathscr{C}(L)$ and $\chi \in \mathscr{C}(K)$ such that $\chi$ is an extension of $\theta$. Trivially, $K R$-generates $B$ and $E=\left\{c_{1}+\cdots+c_{2 n} \mid c_{1}, \cdots, c_{2 n} \in K\right\}$, where $E$ is the ideal of $B$ evenly generated by $L$. Let $I_{\theta}$ and $I_{z}$ be the ideals of $E$ generated by $\{a+b \mid a, b \in L$ and $a \theta b\}$ and $\{c+d \mid c, d \in K$ and $c \chi d\}$ respectively. Clearly $I_{\theta} \subseteq I_{\chi}$. Suppose (by way of contradiction) that $I_{\theta} \neq I_{\chi}$. Let $\Psi \in \mathscr{C}(L)$ such that $(\Psi) g^{-1}=I_{\chi}$, where $g^{-1}$ is the isomorphism given in Theorem 2.8 . Since $I_{x} \supset I_{\theta}$, we have by Lemma 2.6 that there exists $a, b \in L$ such that $a+b \in I_{\chi} \backslash I_{\theta}$ and $(a, b) \in \Psi \backslash \theta$. Now $a+b \in I_{\chi}$ implies $(a, b) \in \chi$ and hence $(a, b) \in \theta$, a contradiction. Therefore $I_{\chi}=I_{\theta}$. Since $K$ generates $B$ and $E$ is the ideal of $B$ evenly generated by $K$, we have by Theorem 2.8 that $\chi$ is unique.

COROLlaRY 4.3. Let $B$ be a generalized Boolean lattice R-generated by $L$.

(i) If $0 \in L$, then $B$ has $(E P)$ over $L$.

(ii) If $0 \notin L$, then $B \backslash E$ has $(E P)$ over $L$.

Note that in the preceding corollary, if $0 \notin L$, then $B / E$ is a relatively complemented lattice without a smallest element. Also, it gives a corrected version of Grätzer (1971; Lemma 5, page 104).

LEMMA 4.4. Let $K$ be a distributive lattice that has (EP) over $L$.

(i) If $a_{0}$ is the smallest element of $L$, then $a_{0}$ is the smallest element of $K$.

(ii) If $c_{0}$ is the smallest element of $K$, then $c_{0} \in L$.

Proof. (i) Let $e \in K$ and $\chi=\left\{(c, d) \mid c, d \in K, c \wedge e \wedge a_{0}=d \wedge e \wedge a_{0}\right.$, and $\left.c \vee a_{0}=d \vee a_{0}\right\}$. It is readily verified that $\chi \in \mathscr{C}(K)$ and $\left(e \wedge a_{0}, a_{0}\right) \in \chi$. If $a, b \in L$ and $a \chi b$, then $a=b$ as $a_{0}$ is zero the of $L$. Therefore $\chi \cap(L \times L)=$ $\{(a, a) \mid a \in L\}$ and since $K$ has $(E P)$ over $L$, it follows that $\chi=\{(c, c) \mid c \in K\}$. Hence $e \wedge a_{0}=a_{0}$.

(ii) If $c_{0} \notin L$, then by (i), $L$ does not have a smallest element. If $D$ is the dual ideal of $K$ generated by $L$, then $D=\{c \mid c \geqq a$ for some $a \in L\}$ and $D \cap\left\{c_{0}\right\}=\square$. 
By Grätzer (1971; Theorem 15, page 75) there exists a prime ideal $P$ of $K$ such that $c_{0} \in P$ and $P \cap \cap D=\square$. Let $\chi=\{(c, d \mid c, d \in P$ or $c, d \in K / P\}$. Then $\chi \in \mathscr{C}(K)$ and $K / \chi=\{P, K \backslash P\}$. Moreover, $\chi \cap(L \times L)=L \times L=(K \times K) \cap(L \times L)$. Thus $K$ does not have $(E P)$ over $L$.

We now prove a converse of Theorem 4.2.

THEOREM 4.5. Let $K$ be a distributive lattice that has (EP) over $L$. Then there exists a generalized Boolean lattice $B$ that is R-generated by L and such that $K$ is a sublattice of $B$. Moreover, if $L$ does not have a smallest element, then $K \subseteq B \backslash E$, where $E$ is the ideal of $B$ evenly generated by $L$.

Proof. Let $C$ be a generalized Boolean lattice $R$-generated by $K$ and let $D=\left\{x \mid x \in C\right.$ and there exists $a_{1}, \cdots, a_{2 n} \in L$ such that $\left.x=a_{1}+\cdots+a_{2 n}\right\}$. Since $L$ is a sublattice of $C, D$ is a subring of $C$. Let $c, d \in K, \chi_{1}=\{(x, y) \mid x, y \in K$ and $x+y \leqq c+d\}$, and $\chi_{2}=\{(x, y) \mid x, y \in K$ and $x+y \leqq e \leqq c+d$ for some $e \in D\}$. Clearly $\chi_{1}$ is reflexive and symmetric. If $(x, y),(y, z) \in \chi_{1}$, then $x+y \leqq c+d$ and $y+z \leqq c+d$. Hence $(x+y) \vee(y+z) \leqq c+d$. Now $x+z=(x+y)+(y+z)$ is the relative complement of $(x+y) \wedge(y+z)$ in $[0,(x+y) \vee(y+z)]$ and hence $x+z \leqq c+d$. Therefore $\chi_{1}$ is an equivalence relation on $K$. Let $(x, y) \in \chi_{1}$ and $z \in K$. Then $x z+y z=(x+y) \wedge z \leqq x+y$ $\leqq c+d$ and so $(x \wedge z, y \wedge z) \in \chi_{1}$. Now $x \wedge z+y \vee z=x+z+x z+y+z$ $+y z=(x+y)+(x+y) z \leqq(x+y) \vee((x+y) z) \leqq x+y \leqq c+d$. Therefore, $(x \vee z, y \vee z) \in \chi_{1}$ and so $\chi_{1} \in \mathscr{C}(K)$. A similar argument yields that $\chi_{2} \in \mathscr{C}(K)$ and obviously $\chi_{2} \subseteq \chi_{1}$. If $(a, b) \in \chi_{1} \cap(L \times L)$, then $a+b \leqq c+d$ and $a+b \in D$. Therefore $(a, b) \in \chi_{2}$. Since $K$ has $(E P)$ over $L$, it follows that $\chi_{1}=\chi_{2}$. Since $(c, d) \in \chi_{1}$, we have $c+d \leqq e \leqq c+d$ for some $e \in D$ so $c+d \in D$. It follows that $D$ is the ideal of $C$ evenly generated by $K$.

If $K$ has a smallest element $c_{0}$, then by Lemma $4.4, c_{0} \in L$ and $c_{0}$ is the zero of $C$. Thus, since $0 \in K$, we have by Theorem 2.4 that $D=C$. Therefore $C$ is a generalized Boolean lattice $R$-generated by $L$ and $D$ is the ideal evenly generated by $L$.

If $K$ does not have a smallest element, then by Lemma 4.4, $L$ does not have a smallest element. Since $K$ generates $C$ and $0 \notin K$, we have by Corollary 2.5 that $D$ is a maximal ideal of index two in $C$ and $K \cap D=\emptyset$. Thus if $a \in L$, $D+a=C \backslash D$. Again we have that $C$ is a generalized Boolean lattice $R$-generated by $L$ and $D$ is the ideal evenly generated by $L$.

Finally, if $L$ does not have a smallest element, then $K$ does not have a smallest element and so $K \subseteq C \backslash D$.

As a corollary to the proof of this theorem we have

Corollary 4.6. Let $K$ be a distributive lattice that has $(E P)$ over $L$. If $C$ is a generalized Boolean lattice R-generated by $K$, then $C$ is $R$-generated by $L$. 
COROLlaRY 4.7. If $K$ is a relatively complemented distributive lattice that has $(E P)$ over $L$, then there exists a generalized Boolean lattice $B$ that is $R$-generated by $L$ and such that $K=B$ or $K=B \backslash E$.

Proof. By the theorem there is a generalized Boolean lattice $R$-generated by $L$ such that $K$ is a sublattice of $B$. If $0 \in L$, then $0 \in K$ and so $K$ is a generalized Boolean lattice. Let $a, b \in L$ and let $c$ be the relative complement of $a \wedge b$ in the interval $[0, a \vee b]_{K}$ of $K$. Then $c=a+b$ and it follows that $E \subseteq K$. By Theorem $2.5, E=B$.

If $0 \notin L$, then by the theorem $K \subseteq B \backslash E$. Let $x \in B \backslash E$. Then $x=a_{1}+\cdots+a_{2 n-1}$ where $a_{1}, \cdots, a_{2 n-1} \in L$ with $a_{1} \leqq \cdots \leqq a_{2 n-1}$. If $n=1$, then $a_{1} \in K$. Suppose that $n>1$ and that $a_{2}+\cdots+a_{2 n-2} \in K$. Now $a_{1} \leqq a_{2} \leqq a_{2}+\cdots+a_{2 n-2} \leqq$ $a_{2 n-2} \leqq a_{2 n-1}$. Let $c$ be the relative complement of $a_{2}+\cdots+a_{2 n-2}$ in the interval $\left[a_{1}, a_{2 n-1}\right]_{K}$ of $K$. Then $a_{1}=c \wedge\left(a_{2}+\cdots+a_{2 n-2}\right)$ and $a_{2 n-1}=$ $c \vee\left(a_{2}+\cdots+a_{2 n-2}\right)=c+a_{2}+\cdots+a_{2 n-2}+c\left(a_{2}+\cdots+a_{2 n-2}\right)$. Hence, $a_{1}+\cdots+a_{2 n-1}=c \in K$.

CoRollary 4.8. Let $K$ be a relatively complemented distributive lattice that has $(E P)$ over $L$. Then no proper sublattice of $K$ contains $L$ and is relatively complemented.

Proof. Let $M$ be a relatively complemented sublattice of $K$ that contains $L$. By the preceding corollary, there is a generalized Boolean lattice $B$ that is $R$-generated by $L$ and such that $K=B$ or $K=B \backslash E$. Then the proof of Corollary 4.7 shows that $M=K$.

COROLlaRY 4.9. Let $K_{1}$ and $K_{2}$ be relatively complemented distributive lattices which have (EP) over $L$. Then there is an isomorphism of $K_{1}$ onto $K_{2}$ that is the identity on $L$.

Proof. Let $B_{1}$ and $B_{2}$ be generalized Boolean lattices that are $R$-generated by $K_{1}$ and $K_{2}$ respectively. By Corollary $4.6, B_{1}$ and $B_{2}$ are $R$-generated by $L$. By Grätzer (1971; Theorem 6, page 104) there is an isomorphism $q$ of $B$ onto $B_{2}$ that is the identity on $L$.) (We note that Theorem 6 of Grätzer (1971) is valid even though Lemma 5 which is invalid, is used in the proof.) If $0 \in L$, then $B_{1}=K_{1}$ and $B_{2}=K_{2}$. If $0 \in L$, then $K_{1}=B_{1} / E_{1}$ and $K_{2}=B_{2} / E_{2}$, where $E_{i}$ is the ideal of $B_{i}$ evenly generated by $L$. Since $\left(K_{1}\right) q$ is a relatively complemented lattice that contains $L$, we ghave by Corollary 4.8 that $\left(K_{1}\right) q=K_{2}$.

\section{An example}

The motivation for many of the ideas in this note is the following example.

Let $P$ denote the power set of the naturally ordered set of integers and $F$ denote the collection of all finite subsets of the integers. For $n \in \mathbb{Z},(n]$ will 
denote the ideal of $\mathbb{Z}$ generated by $n$. Let $B=F \cup\{(n] \cup S \mid n \in \mathbb{Z}$ and $S \in F\}$. Then $B$ is a sublattice of the complete Boolean lattice $P$ and it is readily verified that $B$ is a generalized Boolean lattice.

Let $r$ be the mapping of $\mathscr{C}(\mathbb{Z})$ into $P$ given by $(\theta) r=\mathbb{Z} \backslash\{n \mid n=1$.u.b. $[n] \theta\}$. Then $r$ is an isomorphism of $\mathscr{C}(\mathbb{Z})$ onto $P$ and hence $\mathscr{C}(\mathbb{Z})$ is a complete Boolean lattice. If $L=\{(n] \mid n \in \mathbb{Z}\}$, then $L$ is isomorphic to $\mathbb{Z}, B$ is $R$-generated by $L$, and $F$ is the ideal of $B$ that is evenly generated by $L$. By Corollary $2.11, \mathscr{C}(F)$ is isomorphic to $\mathscr{C}(L)$ and hence to $\mathscr{C}(\mathbb{Z})$. Since $F$ satisfies the descending chain condition, there does not exist an isomorphism of $L$ into $F$.

Suppose (by way of contradiction) that $\mathscr{C}(\mathbb{Z})$ is isomorphic to $\mathscr{C}(B)$. Since $B$ is a generalized Boolean lattice, $\mathscr{I}(B)$ is isomorphic to $\mathscr{C}(B)$. Thus the ideals of $B$ form a Boolean lattice. However, by the corollary to Theorem 4.3 (Hashimoto (1952; page 165)) this implies that $B$ satisfies the descending chain condition. This is impossible as $L$ is a sublattice of $B$.

\section{References}

G. Grätzer (1971), Lattice Theory, (W. H. Freeman and Company, San Francisco, 1971).

G. Grätzer and E. T. Schmidt (1958), 'On the generalized Boolean algebra generated by a distributive lattice', Indag. Math. 20, 54-553.

J. Hashimoto (1953), 'Ideal theory for lattices', Math. Japan. 2, 149-186.

University of Houston

U.S.A.

and

University of Wyoming,

U.S.A. 